\title{
Arterial Access Port Removal
}

National Cancer Institute

\section{Source}

National Cancer Institute. Arterial Access Port Removal. NCI Thesaurus. Code C96175.

Removal of a medical device implanted in an artery for the delivery of medication, fluids, nutrition, or other ordered treatment. 